\title{
TUBERCULOSIS EN PACIENTES CON ARTRITIS REUMATOIDE TRATADOS CON LEFLUNOMIDA. DESCRIPCIÓN DE UNA SERIE DE CASOS.
}

Public a d o:

15 de Abril, 2021 


\section{Autores}

Alexis Javier Atuesta Rodriguez, MD Médico Reumatólogo

Universidad Nacional de Colombia ajatuestar@unal.edu.co

ORCID: 0000-0003-2993-9348

Yimy F. Medina Velásquez, MD

Médico Reumatólogo

Universidad Nacional de Colombia yimy.medina@gmail.com

ORCID: 0000-0003-1255-6703 
ARTíCULO CIENTÍFICO

\section{TUBERCULOSIS IN PATIENTS WITH RHEUMATOID ARTHRITIS TREATED WITH LEFLUNOMIDE. DESCRIPTION OF A SERIES OF CASES}


In patients with rheumatoid arthritis (RA), per se, there is a greater risk of infections, this, the above added to the treatment generates more complications. Leflunomide, an antirheumatic drug, may increase the risk of active tuberculosis in this population, however there is no clarity on this association. The aim of the study is to present a series of cases of RA patients treated with Leflunomide who were screened for tuberculosis. The state of infection in these patients is described.

\section{Methodology}

Patients with RA by ACR / EULAR classification criteria underwent a review of clinical records and the development of active tuberculosis, evaluating variables associated with RA. A subgroup analysis was done based on whether they had latent tuberculosis or not. The results are those of a series of cases of patients treated with Leflunomide in RA.

\section{Results}

A case of active tuberculosis was identified in the subgroup of latent tuberculosis, in the other subgroup without this condition, no cases were found. More than $75 \%$ of the patients were on concomitant therapy with corticosteroids or methotrexate, with a mean age of 60 years and a duration of RA greater than 10 years.

\section{Conclusion}

A series of cases of RA patients treated with Leflunomide in which the possibility of tuberculosis was explored is presented. The search for factors associated with this infectious disease may be of importance in identifying patients at risk for tuberculosis and determining the role of this drug. 


\section{Resumen}

\section{En Español}

En pacientes con artritis reumatoide (AR), per se, existe un riesgo mayor de infecciones, lo cual sumado al tratamiento genera más complicaciones. Leflunomida, un fármaco antirreumático, puede aumentar el riesgo de tuberculosis activa en esta población; sin embargo, no hay claridad acerca de esta asociación. El objetivo de este estudio es presentar una serie de casos de pacientes con AR en tratamiento con leflunomida en quienes se exploró para tuberculosis. Se describe el estado de la infección en estos pacientes.

\section{Metodología}

A los pacientes con artritis reumatoide, por criterios clasificatorios ACR/EULAR, se les hizo una revisión de los registros clínicos y el desarrollo de tuberculosis activa, evaluando variables asociadas a la AR. Se hizo un análisis por subgrupos teniendo como criterio de selección si tenían tuberculosis latente o no. Los resultados son los de una serie de casos de pacientes tratados con leflunomida en AR.

\section{Resultados}

Se identificó un caso de tuberculosis activa en el subgrupo de tuberculosis latente; en el otro subgrupo sin esta condición no se encontraron casos. En un porcentaje mayor, el 75 \% de los pacientes estaba en terapia concomitante con corticoesteroides o metotrexate, con media de edad de 60 años y una duración de la AR mayor a 10 años.

\section{Conclusión}

Se presenta una serie de casos de pacientes con AR tratados con leflunomida en quienes se exploró la posibilidad de tuberculosis. La búsqueda de factores asociados a esta enfermedad infecciosa puede ser de importancia para identificar a los pacientes en riesgo de tuberculosis y determinar la incidencia de este fármaco. 


\section{Resumen En Portugués}

Em pacientes com artrite reumatoide (AR), por si só, existe um risco maior de infecções, isso, o exposto acima adicionado ao tratamento gera mais complicações. A leflunomida, um medicamento antirreumático, pode aumentar o risco de tuberculose ativa nessa população, porém não há clareza sobre essa associação. O objetivo do estudo é apresentar uma série de casos de pacientes com AR tratados com Leflunomida que foram testados para tuberculose. O estado da infecção nesses pacientes é descrito.

\section{Metodologia}

Pacientes com AR pelos critérios de classificação ACR / EULAR foram submetidos a revisão de prontuários e desenvolvimento de tuberculose ativa, avaliando variáveis associadas à AR. Uma análise de subgrupo foi feita com base no fato de eles terem tuberculose latente ou não. Os resultados são de uma série de casos de pacientes tratados com Leflunomida na AR.

\section{Resultados}

Um caso de tuberculose ativa foi identificado no subgrupo de tuberculose latente, no outro subgrupo sem essa condição não foram encontrados casos. Mais de $75 \%$ dos pacientes estavam em terapia concomitante com corticosteroides ou metotrexato, com idade média de 60 anos e duração da AR superior a 10 anos.

\section{Conclusão}

É apresentada uma série de casos de pacientes com AR tratados com Leflunomida nos quais foi explorada a possibilidade de tuberculose. A busca de fatores associados a essa doença infecciosa pode ser importante na identificação de pacientes com risco para tuberculose e na determinação do papel dessa droga. 


\section{Introducción}

La artritis reumatoide (AR) es una enfermedad autoinmune de carácter crónico que afecta aproximadamente al $1 \%$ de la población mundial. El tratamiento, por lo general, se basa en la administración de fármacos antirreumáticos modificadores de la enfermedad (FARME); en ocasiones, según la actividad, se requiere la combinación de medicamentos e, incluso, hasta el uso de terapias biológicas, con el consecuente aumento en el riesgo de eventos adversos (EA) (1).

Los eventos adversos en AR se presentan tanto inherentes a la patología como asociados al tratamiento empleado para el control de la enfermedad $(1,2)$. Se ha identificado un aumento en el riesgo de infecciones de pacientes con AR, en relación con la patología y el uso de ciertos medicamentos indicados en el tratamiento de la enfermedad, lo que conlleva a más complicaciones (3). Estos fármacos van desde los corticoesteroides, con mayor riesgo según la dosis-día y la dosis acumulada, pasando por los FARME sintéticos, como el metotrexate y la leflunomida, hasta terapias biológicas, como los inhibidores del TNF y otros agentes con diferentes blancos de acción. Todos los anteriores fármacos están asociados con aumento en el riesgo de infecciones fundamentalmente bacterianas $(4,5)$.

La leflunomida es un fármaco usado ampliamente con éxito en el tratamiento de la artritis reumatoide, y es recomendado tanto en monoterapia como en combinación (6). Su perfil de seguridad incluye efectos que van desde complicaciones dermatológicas, hepáticas, hematológicas, hasta un aumento en el riesgo de infecciones por gérmenes oportunistas, como neumonía por Pneumocystis Jirovecii, Aspergillosis; además, se ha descrito una asociación con la reactivación de tuberculosis (TB) latente, con afectación pulmonar y manifestaciones extrapulmonares (7). El riesgo de infección es mayor si se suma al uso de otros fármacos inmunosupresores, o si el paciente presenta otras comorbilidades, como diabetes mellitus, cáncer, entre otras. No obstante, otros efectos adversos, como elevación de enzimas hepáticas, intolerancia gastrointestinal, alopecia y neutropenia, resultan más frecuentes como motivos de suspensión de la terapia con leflunomida. (8) Ciertas revisiones han mostrado una prevalencia de hasta $6 \%$ de TB activa con relación al consumo de esta medicación; sin embargo, existe controversia en diferentes publicaciones acerca de si se debe realizar en todos los pacientes con AR una búsqueda de tuberculosis latente antes de iniciar tratamiento con leflunomida $(2,9)$. El objetivo de este estudio es presentar los hallazgos en una serie de casos de pacientes con artritis reumatoide, tratados con leflunomida, y la presencia de reactivación de TB en esta población. 


\section{Material y Métodos}

Se presenta un estudio observacional retrospectivo de revisión de casos de pacientes con artritis reumatoide en tratamiento con leflunomida, como parte del manejo FARME. Previamente se le realizaron a los pacientes estudios de laboratorio e imágenes con el fin de identificar TB activa y TB latente. Se fraccionó la corte en dos grupos: Pacientes con presencia de TB latente, evaluado por prueba de derivado proteico purificado (PPD, por sus siglas en inglés) positiva, dado por induración mayor a $5 \mathrm{~mm}$ a las 72 horas, con una radiografía de tórax negativa. El otro grupo estuvo integrado por pacientes con PPD negativa y radiografía de tórax negativa. Para identificar el diagnóstico de TB activa se hizo una búsqueda en los registros clínicos de los pacientes; también se procedió a: evaluación clínica con base en los síntomas respiratorios; diagnóstico microbiológico, como coloración ácido alcohol resistente en esputo o cultivo para M. tuberculosis; o diagnóstico por imágenes de tórax, según la interpretación del radiólogo. Se identificaron variables de interés asociadas con la probabilidad de reactivación de la enfermedad. Se presentan los resultados en frecuencias relativas y absolutas, tanto para el resultado primario como para las variables evaluadas en relación. En este trabajo solo se presentan los datos clínicos y paraclínicos de los pacientes incluidos en porcentajes, sin hacer análisis de asociación.

\section{Resultados}

En una institución de alto nivel de complejidad se identificaron, en la consulta de reumatología, un total de 26 pacientes con diagnóstico de artritis reumatoide, según los criterios clasificatorios 1987 o ACR/EULAR 2010 (10,11). Se muestran en este trabajo los hallazgos en dos subgrupos: el primero presentaba el diagnóstico de tuberculosis latente $\mathrm{o}$ activa $(\mathrm{n}=5$ pacientes) y el segundo presentaba tuberculosis latente negativa ( $\mathrm{n}=21$ pacientes); lo anterior teniendo en cuenta la premisa de otros estudios, los cuales indican que los pacientes con TB latente pueden tener un mayor riesgo de reactivación de la enfermedad con leflunomida. Luego de hacer el análisis microbiológico (en pacientes sintomáticos) por baciloscopia o cultivo, y radiografía de tórax, independientemente de síntomas, se identificó solo un caso de TB activa en el grupo con tuberculina positiva. En la tabla 1 se presentan las frecuencias de presentación de variables propias de los pacientes, como edad, sexo, y también aquellas asociadas a otros marcadores clínicos y paraclínicos. 


\section{Tabla 1: Frecuencias de presentación de variables en pacientes con tuberculina positiva y negativa}

\begin{tabular}{|l|l|l|}
\hline VARIABLE & Tuberculina positiva $\mathbf{n = 5}(\%)$ & Tuberculina negativa $\mathbf{n = 2 1}(\%)$ \\
\hline Edad (media años) & 66 & 60 \\
\hline Femenino & $4(80)$ & $20(95)$ \\
\hline Duración de AR (media años) & 21 & 14 \\
\hline Tuberculosis activa & $1(20)$ & $\mathbf{0}$ \\
\hline Tabaquismo activo & $3(60)$ & $8(38)$ \\
\hline $\begin{array}{l}\text { Tratamiento } \\
\text { Corticoesteroide (CE) }\end{array}$ & $5(100)$ & $19(90)$ \\
\hline Dosis acumulada año CE (g) & 2,05 & 2,1 \\
\hline Diabetes mellitus & $1(20)$ & $6(28)$ \\
\hline Factor reaumatoide positivo & $4(80)$ & $20(95)$ \\
\hline Anticuerpos CCP positivos & $4(80)$ & $18(85)$ \\
\hline Anticuerpos antinucleares & $3(60)$ & $11(52)$ \\
\hline Tratamiento metotrexato & $3(60)$ & $12(58)$ \\
\hline
\end{tabular}

Frecuencias de presentación de variables en pacientes con tuberculina positiva y negativa.

Se presentan las frecuencias de presentación de variables tanto propias de los pacientes como edad, sexo y aquellas asociadas a otros marcadores clínicos y paraclínicos 


\section{Discusión}

Leflunomida es un fármaco antirreumático modificador de la enfermedad que se ha usado ampliamente con éxito en el tratamiento de la artritis reumatoide (AR) desde hace más de 20 años. Su efecto positivo radica en las propiedades antinflamatorias e inmunomoduladoras, a través de su metabolito activo teriflunomida que inhibe la síntesis de novo de nucleótidos de pirimidina y, al mismo tiempo, es responsable de inhibir enzimas tipo tirosina cinasa, a cargo de la señalización intracelular en células T y B, con la subsecuente disminución en la respuesta proinflamatoria de predominio $\mathrm{TH} 1$ y $\mathrm{TH} 17$, donde moléculas con el factor de necrosis tumoral (TNF), IFN-y, IL-12, IL-15 son de gran importancia en la AR $(2,11)$.

Una actividad regulada de estas moléculas es crucial en la respuesta inmune contra patógenos de tipo bacterianos, entre los cuales el Mycobacterium tuberculosis es uno de los más representativos, pues es un germen intracelular que tiene la capacidad de permanecer en un estado latente y presentar una reactivación con fármacos que pueden afectar la respuesta inmune usual.

El uso de leflunomida en monoterapia o en combinación con otros fármacos, como metotrexate y corticoesteroides, se ha incrementado en pacientes con artritis reumatoide con una actividad moderada o alta como una buena alternativa antes de considerar una terapia biológica, lo cual permite una mejoría clínica favorable a un menor costo. De hecho, se ha demostrado que la combinación de FARME sintéticos presenta una respuesta similar al uso de rituximab a bajas dosis, y no se registra un aumento en los casos de infecciones graves, ni tampoco eventos adversos de consideración (12). Por lo tanto, resulta una alternativa en regiones donde es limitada la disponibilidad de otras terapias modificadoras de la enfermedad. El riesgo de reactivación de tuberculosis en pacientes tratados con metotrexate y leflunomida resulta ser menor al $1 \%$ en algunos registros, lo cual permite implementar una terapia segura $(13,14,15)$.

En Colombia, el 24 de noviembre de 2016, el INVIMA (Instituto Nacional de Vigilancia de Medicamentos y Alimentos) socializó información de sus bases de datos, y también las recomendaciones publicadas por la Agencia Nacional de Vigilancia Sanitaria de Brasil (ANVISA), respecto a que en los pacientes que se encuentran bajo tratamiento con leflunomida existe la posibilidad de activación o reactivación de tuberculosis. Otras sociedades, como la EMA (European Medicines Agency) y la FDA (Food and Drug Administration) también plantean un mayor riesgo para el desarrollo de tuberculosis; sin embargo, no se menciona una clara recomendación en cuanto a la búsqueda activa de tuberculosis en pacientes próximos a ser 
medicados o que ya están en tratamiento con leflunomida $(16,17,18,24)$. En las recomendaciones del Colegio Americano de Reumatología (ACR) se plantea suspender leflunomida en casos de infección bacteriana activa, incluyendo tuberculosis, a menos que sea un estado latente una vez se inicie el tratamiento (12).

En un estudio colombiano, que evaluó los efectos adversos asociado a terapia FARME sintética en pacientes con AR, se encontró una incidencia para leflunomida de 4,9 eventos adversos por cada 100 pacientes/año, imperando los casos de eventos gastrointestinales y hepatitis, sin un claro riesgo para el incremento de infecciones, tanto para monoterapia como para terapia combinada con metotrexate $(19,20)$. No obstante, en otros reportes se plantea una asociación significativa, con un OR de 6,02 (IC 1.47-24.65) para reactivación de tuberculosis en pacientes expuestos a leflunomida (21). Adicionalmente, existen reportes de casos en los que se menciona una asociación de reactivación para micobacterias no tuberculosas (22).

El riesgo de tuberculosis activa en este grupo poblacional está sujeto no solo al tratamiento farmacológico, sino también a otros factores asociados propios del paciente, tales como: antecedente de neumopatía crónica independiente de la etiología, tabaquismo, inmunodeficiencias primarias o adquiridas, entre otras $(7,19)$. Estudios observacionales señalan que el riesgo de desarrollo de tuberculosis con leflunomida y metotrexate está aumentado en población mayor a 65 años, llegando hasta 4 veces con respecto a pacientes sin estas terapias, especialmente en países con una mayor prevalencia de la enfermedad (23). A pesar de que en otras revisiones se ha identificado y propuesto la leflunomida como agente responsable de la reactivación de la enfermedad, en este grupo de pacientes solo se encontró un caso de tuberculosis activa en el subgrupo con TB latente; y hay que tener en consideración que el paciente con reactivación de su patología infecciosa presentaba variables de riesgo, tales como estar en terapia inmunosupresora con tratamiento biológico tipo anti TNF, tener una evolución del AR mayor a 10 años y ser exfumador.

Lo anterior puede sugerir que quizás no a todos los pacientes susceptibles de iniciar tratamiento con leflunomida se les debería hacer búsqueda activa de tuberculosis activa o latente. Sin embargo, existen otros grupos de mayor riesgo en los que sí se deben considerar estudios previos de imágenes y también puede ser indicado la búsqueda de tuberculosis latente, sobre todo en aquellos habitantes de zonas endémicas para la enfermedad. De igual manera es recomendable la búsqueda de TB latente en personas que tengan residencia con este diagnóstico, antecedentes de neumopatía crónica o hallazgos en imágenes que pueden sugerir enfermedad activa; así mismo, se 
deben hacer estudios en pacientes en terapia concomitante con esteroides a dosis moderada a alta, y tratamiento concomitante con otras terapias inmunomoduladoras, biológicas y no biológicas $(2,11)$.

En las recomendaciones del ACR no hay una clara sugerencia acerca de una búsqueda para tuberculosis antes del inicio de terapia con metotrexate o leflunomida; sin embargo, sí existen otras sociedades de reumatología que recomiendan esta práctica de preferencia para metotrexate. Si bien la recomendación, en cuanto a la tamización para tuberculosis en pacientes que van a iniciar tratamiento con FARME sintéticos es discordante por lo descrito en diferentes reportes, se debe hacer una individualización para cada caso, pues si presentan una clínica o imágenes sugestivas de la enfermedad es obligatorio la búsqueda para tuberculosis, sobre todo por el impacto que puede tener en complicaciones y en la supervivencia de los pacientes con AR $(12,23)$.

Las limitaciones de este trabajo se basan en que se circunscribe solo a un estudio observacional de revisión de una serie de casos. Sin embargo, este tipo de trabajos puede jugar un papel protagónico en la detección de epidemias al informar acerca de uno o varios casos; o registrar brotes de alguna enfermedad nueva, o que previamente era conocida y que estuviese controlada. También son útiles para describir las características de enfermedades muy poco frecuentes, y pueden convertirse en el paso inicial para su posterior investigación $(25,26)$.

Los hallazgos encontrados en nuestra serie deben ser evaluados en un estudio analítico en el que se logren controlar las variables de confusión y se desarrolle un modelo de asociación y multivariado. De esta manera se podrá identificar cuál es el riesgo real de tuberculosis con una causa identificada por leflunomida, y a partir de ello generar una recomendación sólida sobre la tamización en estos pacientes.

\section{Conclusión}

Se presenta una serie de casos de pacientes con AR en tratamiento con leflunomida, en los que se exploró la presencia de tuberculosis, tanto en su forma activa como latente. La búsqueda de otros factores asociados a esta enfermedad infecciosa puede ser de importancia para identificar a los pacientes en riesgo de desarrollar una forma sintomática de tuberculosis. No obstante, este trabajo no permite entregar una recomendación contundente en cuanto a la influencia de la leflunomida y la predisposición a tuberculosis. Se requieren estudios de mejor calidad metodológica para poder responder esta pregunta, sobre todo en nuestro medio donde la tuberculosis es aún una enfermedad de importancia en salud pública. 


\section{Referencias}

1. Agrawal S, Sharma A. Dual mycobacterial infection in the setting of leflunomide treatment for rheumatoid arthritis. Ann Rheum Dis. 2007;Feb;66(2):277.

2. Hocevar A, Rozman B, Praprotnik S, et al. Leflunomide-associated tuberculosis? Rheumatology 2006;45:228-243

3. Carmona L, Hernández-García C, Vadillo $C$ et al. Increased risk of tuberculosis in patients with rheumatoid arthritis. J Rheumatol 2003;30:1436-9.

4. Bieber J, Kavanaugh A. Consideration of the risk and treatment of tuberculosis in patients who have rheumatoid arthritis and receive biologic treatments. Rheum Dis Clin North Am 2004;30:257-70.

5. Jenks K, Stamp L, O'Donnell J, et al. Leflunomide-associated infections in rheumatoid arthritis. J Rheumatol 2007;Nov;34(11):2201-3.

6. Cannon GW, Kremer JM. Leflunomide. Rheum Dis Clin North Am 2004;30:295-309.

7. Doran MF, Crowson CS, Pond GR, O'Fallon M, Gabriel SE. Frequency of infection in patients with rheumatoid arthritis compared with controls. A population-based study. Arthritis Rheum 2002;46:2287-93.

8. Monteiro A, Pires C, Vargas-Santos AB, et al. Causes of synthetic disease-modifying drugdiscontinuation in rheumatoid arthritis: Data from a large real-life cohort. PLoS One. 2019 Mar 1;14(3):e0213219
9. Dixon WG, Watson K, Lunt M, et al. Rates of serious infection, including site-specific and bacterial intracellular infection, in rheumatoid arthritis patients receiving anti-tumor necrosis factor therapy. Results from the British Society for Rheumatology Biologics Register. Arthritis Rheum 2006;54:2368-76.

10. Aletaha D, Neogi T, Silman A, et al. 2010 Rheumatoid arthritis classification criteria: an American College of Rheumatology/European League Against Rheumatism collaborative initiative. Arthritis Rheum 2010 Sep;62(9):2569-81.

11. Manna SK, Mukhopadhyay A, Aggarwal BB. Leflunomide suppresses TNF-induced cellular responses: effects on NF-B, activator protein-1, c-Jun N-terminal protein kinase and apoptosis. J Immunol 2000;165:5962-9

12. Singh, J.A., Saag, K.G., Louis Bridges,et al. 2015 American College of Rheumatology Guideline for the Treatment of Rheumatoid Arthritis. Arthritis Care Res. (Hoboken). 68, 1-26

13. Hodkinson B, Ross K, Tikly M. Combination leflunomide and methotrexate in refractory rheumatoid arthritis: a biologic sparing approach. Ther Adv Musculoskelet Dis 2016 Oct;8(5):172-179.

14. Wijesinghe H, Galappatthy P, Rajiva de Silva, et al. Leflunomide is equally efficacious and safe compared to low dose rituximab in refractory rheumatoid arthritis given in combination with methotrexate: results from a 
randomized double blind controlled clinical trial. BMC Musculoskelet Disord. 2017;Jul 19;18(1):310.

15. Doran MF, Crowson CS, Pond GR, et al. Predictors of infection in rheumatoid arthritis. Arthritis Rheum 2002 Sep;46(9):2294-300

16. Ministerio de salud - Invima. Dirección de medicamentos y productos biológicos grupo de farmacovigilancia - Leflunomid. Información para profesionales de la salud. 2016 [citado 24 noviembre 2016]. Disponible en: http://app.invima.gov.co/alertas/ckfinder/userfiles/files/IN-

FORMES\%20DE\%20SEGURIDAD/Medicamentos/2016 /-/Noviembre/INFORMACION-PARA-PROFESIONALESDE-LA-SALUD-PACIENTES-Y-CUIDADORES-LEFLUNOM IDA.pdf

17. Anvisa AN de VS-. Centro de vigilancia sanitária [Internet]. Download Alerta Terapêutico - Leflunomide. 2012 [citado 20 marzo 2012]. Disponible en: http://www.cvs.saude.sp.gov.br/zip/Alerta\%20Terap\%C3\%AAutico\%2002_01\%20LEFLUNOMIDE.pdf

18. EMA. Summary of Product Characteristics [Internet]. Leflunomide. 2001 [citado 20 marzo 2021]. Disponible en: https://www.ema.europa.eu/en/documents/product-information/le-

flunomide-ratiopharm-epar-product-information_en.pdf

19. Machado-Alba JE, Ruiz, AF, Machado-Duque ME. Adverse drug reactions associated with the use of disease-modifying anti-rheumatic drugs in patients with rheumatoid arthritis. Rev Panam Salud Publica 2014;36(6).

20. Bird P, Griffiths H, Tymms K, et al. The SMILE study -- safety of methotrexate in combination with leflunomide in rheumatoid arthritis. J Rheumatol

2013;Mar;40(3):228-35
21. Sundbaum J. Arkema E, Bruchfeld J, et al. Tuberculosis in biologic-naive patients with rheumatoid arthritis - risk factors and tuberculosis characteristics. Abstract eular jun 2020, sat0069. DOI:10.1136/annrheumdis-2020-eular.5339.

22. Winetsky E. Myers J, Schulz S, et al. A Case of Cavitary Pulmonary Mycobacterium Avium Intracellulare Infection in the Setting of Leflunomide Treatment.J Clin Rheumatol 2017;Jun;23(4):231-232.

23. Brassard P, Lowe AM, Bernatsky S, et al. Rheumatoid arthritis, its treatments, and the risk of tuberculosis in Quebec, Canada. Arthritis Rheum 2009;Mar, 15;61(3):300-4.

24. FDA UF and DA. Contraindications and Warnings [Internet]. Drugsatfda ARAVA. 2011 [citado 1 julio 2011]. Disponible en: https://www.accessdata.fda.gov/drugsatfda_docs/label/2011/020905s022lbl.pdf.

25. F M Abu-Zidan 1, A K Abbas, A F Hefny. Clinical "case series": a concept analysis. Afr Health Sci 2012;4:557-62.

26. Celeste RK. Case Reports and Case Series in the Era of Evidence - Based Medicine. Brazilian. J videoendoscopic surgey 2010;3(2):063-6. 
GLOBAL RHEUMATOLOGY 